\title{
Effect of some parameters on the thermo- hydraulic characteristics of a channel heat exchanger with corrugated walls
}

Houari AMEUR, Djamel SAHEL

DOI: 10.30464/jmee.2019.3.1.53

Cite this article as:

Ameur H., Sahel D. Effect of some parameters on the thermo-hydraulic characteristics of a channel heat exchanger with corrugated walls. Journal of Mechanical and Energy Engineering, Vol. 3(43), No. 1, 2019, pp. 53-60.

\section{VOLUME 3(43) | No. 1 | APRIL 2019} ISSN 2544-0780 | e-ISSN 2544-1671

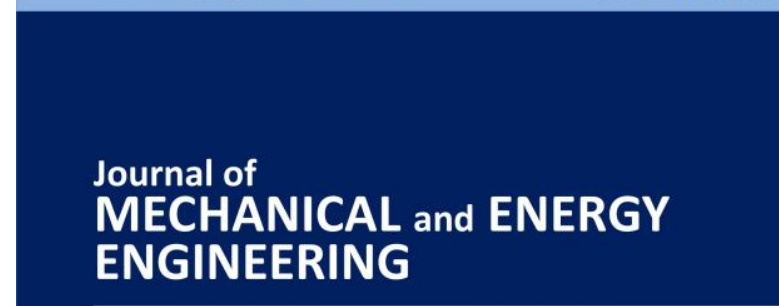

Editor-in-Chief

Waldemar Kuczyński

Editors

Krzysztof Rokosz | Krzysztof Nadolny
Journal of Mechanical and Energy Engineering

Website: jmee.tu.koszalin.pl

ISSN (Print): 2544-0780

ISSN (Online): 2544-1671

Volume: 3(43)

Number: 1

Year: 2019

Pages: 53-60

\section{Article Info:}

Received 24 January 2019

Accepted 18 February 2019

\section{Open Access}

This article is distributed under the terms of the Creative Commons Attribution 4.0 (CC BY 4.0) International License (http://creativecommons.org/licenses/by/4.0/), which permits unrestricted use, distribution, and reproduction in any medium, provided you give appropriate credit to the original author(s) and the source, provide a link to the Creative Commons license, and indicate if changes were made. 


\title{
EFFECT OF SOME PARAMETERS ON THE THERMO-HYDRAULIC CHARACTERISTICS OF A CHANNEL HEAT EXCHANGER WITH CORRUGATED WALLS
}

\author{
Houari AMEUR ${ }^{1 *}$, Djamel SAHEL ${ }^{2}$ \\ ${ }^{1 *}$ Department of Technology, Institute of Science and Technology, University Center of Naama \\ (Ctr Univ Naama), Po. Box 66, 45000, Algeria, e-mail: houari_ameur@yahoo.fr \\ ${ }^{2}$ Department of Technical Sciences, University Amar Thilidji of Laghouat, Algeria
}

(Received 24 January 2019, Accepted 18 February 2019)

\begin{abstract}
The present work is a numerical modeling of the flow of a viscous fluid provided with convective heat transfer in a rectangular channel. We focus on the influence of the shape of corrugations which are present on the channel walls. Three shapes of corrugations are studied and which are: the rectangular, triangular and semi-circular shape. Also, effects of the height and number of corrugations on the fluid dynamics and thermal characteristics of the exchanger are highlighted. The working fluid is non-Newtonian (having a shear thinning behavior modeled by the Otswald law). The obtained results reveal that the presence of corrugations yields a major change in the heat exchange execution. In terms of superiority of heat transfer ratio, the cases under investigation can be classified as follows: rectangular > triangular > semi-circular. However and in terms of low pressure drop, this ranking is reversed. Furthermore, the triangular and semicircular shapes were found to have almost similar effects.
\end{abstract}

Keywords: heat exchanger, corrugated walls, laminar flow, complex fluids

\section{INTRODUCTION}

Heat exchangers (HEs) are employed in various systems such as power plant, heat recovery units and nuclear reactors. The development, reliability and maintainability of these equipment are highly demanded to improve the thermal energy transfer from two or more mediums. Various types of HEs are available, such as plate heat exchangers (flat, brazed or corrugated) [1, 2], shell and tube (horizontal or vertical) [3], micro-channels $[4,5]$ and tubular heat exchangers [6].

In the last decades, the channels with corrugated surfaces are extensively employed in heat exchangers [7]. The corrugated design enhances the fluid mixing and the heat transfer through the channel. However, the pressure drop penalty remains a great challenge $[8,9]$.

Some works have been achieved on the subjects, among others, Rush et al. [10] investigated the thermo-hydraulic characteristics of a sinusoidal wavy channel and they found that the flow rate and channel configuration have a great influence on the position of the onset of mixing and heat transfer rate. For both sinusoidal and arc-shaped channels, Bahaidarah et al. [11] reported that the increase of Reynolds number yields a rise in the heat transfer ratio. For rectangular ducts, the increase of corrugation height may intensify the turbulence on the axe of corrugation, resulting thus in enhanced heat transfer [12]. Also, the increase of wavy amplitude-wavelength ratio yields an increase in the local Nusselt number in the converging section of the channel [13]. For a Reynolds number ranging between 600 and 2000, the results obtained by Blomerius and Mitra [14] concerning the effect of angle between corrugations and the main flow direction reveal that the best performance may be obtained with the configuration of $45^{\circ}$ angle. Ali and Hanaoka [15] studied the forced convective heat transfer of air laminar flow through a V-corrugated channel heated by radiation. They found that the increased inclination angle from $0^{\circ}$ to $60^{\circ}$ yielded an increase in $N u$ from $33 \%$ to $67.3 \%$. The results given 
by Mohammed et al. [16] for a wavy channel reveal an increase in pressure drop with rising wavy angle and a decrease with respect to the channel height. Under turbulent conditions of air flows, Taymaz et al. [17] studied the forced convective heat transfer in a periodic converging-diverging channel. They found that $\mathrm{Nu}$ increases with increased corrugation angle and it decreases with increased corrugation height.

One of the most promising devices for enhancing the thermo-hydraulic performance in micro-turbine recuperators is the Cross-Wavy (CW) surface heat exchanger. Ma et al. [18] studied by experiments and simulations the efficiency of $\mathrm{CW}$ channels in a wind tunnel and they reported that the entrance region has a small influence on the heat transfer coefficient of whole CW channels. Wang et al. [19] studied numerically the influence of geometrical parameters on the performance of corrugated-undulated (CU) heat transfer plates. Due to the drag effect between the main flows in CU channels, intensive secondary flows may occur, and consequently, the cold flow in the center of the passage may be moved to the surfaces and the bottoms of the plates. These authors found also that the U-plate height has a pronounced effect on the disturbance than the U-plate pitch.

For air flowing in grooved tubes (rectangular, trapezoidal and circular) under turbulent conditions $\left(R e=38 \times 10^{3}\right)$, Bilen et al. [20] found an enhancement of heat transfer by about $47 \%, 58 \%$ and $63 \%$ for a rectangular, trapezoidal and circular groove, respectively. Jialing et al. [21] investigated numerically the thermal performance of corrugated, nodal and horizontal grain tubes. Compared to the straight tube, these three shapes revealed an enhancement in heat transfer by 2.31-2.53, 1.18-1.86 and 1.02-1.31 times, but accompanied with higher drag coefficient estimated as: 6.10-7.02, 2.06-11.03 and $0.53-1.83$, respectively.

Elshafei et al. [22] studied the effect of channel spacing and phase shift on the performance of corrugated channels. Compared to parallel plate channel, the obtained results shown an enhancement by a factor of 1.9 to 2.6 for the pressure drop and from 2.6 to 3.2 for the heat transfer coefficient. These authors reported also that the increased channel spacing and phase shift yields an increase in friction factor. Gao et al. [23] interested to a channel with one corrugated wall. The bottom wall of channel has sinusoidal corrugations in the streamwise and spanwise directions. With the rise of wave amplitude, these authors illustrated the separation of flow bubbles and the formation of vortices near the wall streamwise. Zhang and Che [24] studied the influence of corrugation profile (sinusoidal, trapezoidal, elliptic, rectangular and isosceles triangular), corrugation angle and Reynolds number on the efficiency of crosscorrugated channels. Higher values for the friction factor and Nusselt number by about 1-4 times were observed for the trapezoidal plate compared to the elliptic plate. They reported that the small inclination angle and the smooth corrugation shapes are the optimal structures among the cases studied. For a Reynolds number ranging between 400-1400, Akbarzadeh et al. [25] studied the effect of corrugation profile (sinusoidal, trapezoidal and triangular) on the entropy generation and thermo-hydraulic performance of a wavy channel. From the cases studied, the sinusoidal corrugated channels is recommended to be used for their low entropy generations and high efficiency.

Our literature review reveals that the cooling of shear thinning fluids by using heat exchangers with corrugated walls still requires further studies. So, our main objective here is to determine the flow fields and thermal characteristics of shear thinning fluids flowing through rectangular channels with corrugations. Effects of the shape of corrugations (semi-circular, rectangular and triangular shapes) are explored.

\section{PRESENTATION OF THE PROBLEM}

The geometry of the problem under investigation is given in Fig. 1. It concerns a rectangular channel having corrugations of the upper and lower walls. The length of channel $(L)$, its width $(a)$, its height $(b)$ and the length $(l)$ of corrugations are: $300 \mathrm{~mm}, 20 \mathrm{~mm}, 20$ $\mathrm{mm}$ and $20 \mathrm{~mm}$, respectively.

The working fluid is the CMC solution (CarboxyMythl-Cellulose) which has the following rheological properties: consistency index $m=0.002 \mathrm{~Pa} \mathrm{~s}{ }^{n}$, power law index $n=0.8$ and density $\rho=997 \mathrm{~kg} / \mathrm{m}^{3}$. The behavior of this fluid is shear thinning and it is modeled by the Ostwald's model. The inlet temperature of hot fluid is $65^{\circ} \mathrm{C}$ and the temperature at the channel walls is $-5^{\circ} \mathrm{C}$. The left and right surfaces of channel are supposed to be symmetric.

For $h / a=0.3$, three geometric configurations are realized and compared with the smooth channel to investigate the effect of wall shape (Fig. 2); it concerns the circular, triangular and rectangular shapes. Also, the effect of the corrugation height is investigated $(h / a=0.2,0.3$ and 0.4$)$.

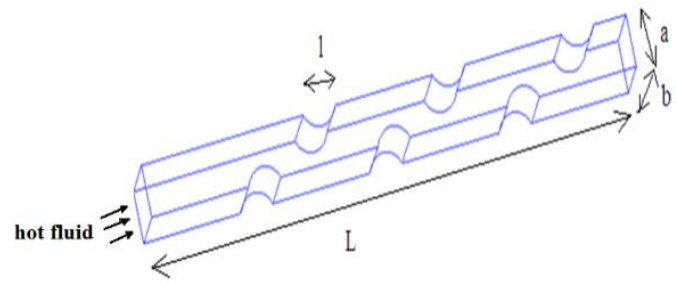

Fig. 1. Geometry of the problem studied 


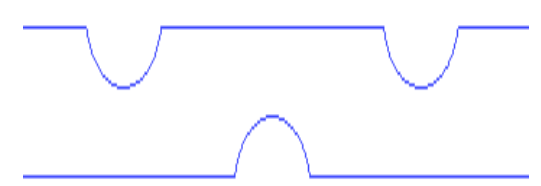

Circular corrugations

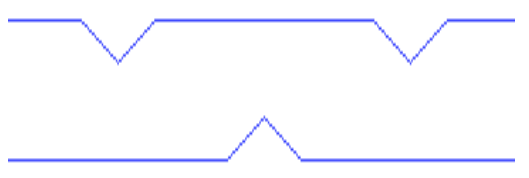

Triangular corrugations

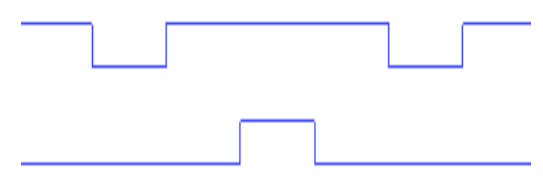

Rectangular corrugations

Fig. 2. The different cases studied

\section{GOVERNING EQUATIONS}

The flow in the channel is continuous, laminar and steady state and the heat transfer is natural. The working fluid has a shear thinning behavior modeled by the Ostwald law.

$$
\begin{gathered}
\tau=m \gamma^{n}, \\
\eta=m \gamma^{n-1}
\end{gathered}
$$

where $\tau$ is the shear stress, $\gamma$ is the shear rate and $\eta$ is the apparent viscosity.

Based on the above assumptions, the governing equations are written as:

- Conservation of mass:

$$
\frac{\partial}{\partial x_{i}}\left(\rho u_{\mathrm{i}}\right)=0
$$

- Conservation of momentum:

$$
\frac{\partial}{\partial x_{\mathrm{i}}}\left(\rho u_{\mathrm{i}} u_{\mathrm{k}}\right)=\frac{\partial}{\partial x_{\mathrm{i}}}\left(\mu_{\mathrm{a}} \frac{\partial u_{\mathrm{k}}}{\partial x_{\mathrm{i}}}\right)-\frac{\partial p}{\partial x_{\mathrm{k}}}
$$

- Conservation of energy:

$$
\frac{\partial}{\partial x_{\mathrm{i}}}\left(\rho u_{\mathrm{i}} T\right)=\frac{\partial}{\partial x_{\mathrm{i}}}\left(\frac{k_{\mathrm{f}}}{C_{\mathrm{p}}} \frac{\partial T}{\partial x_{\mathrm{i}}}\right)
$$

where $C_{\mathrm{p}}$ is the specific heat of working fluids.
For a shear thinning fluid, the Reynolds number is given by:

$$
\operatorname{Re}_{g}=\frac{\rho U^{2-n} D_{h}^{n}}{m}\left(\frac{3 n+1}{4 n}\right)^{-n} 8^{1-n},
$$

where $D_{\mathrm{h}}$ is the hydraulic diameter.

\section{COMPUTATIONAL PROCEDURE}

All geometries were created with the computer tool ICEM CFD (version 18.0). Then, the CFD code (CFX, version 18.0) was employed to achieve simulations of fluid flows and heat transfer phenomena in the heat exchanger channels. With ICEM, unstructured mesh grid consisting of tetrahedral elements was used. To facilitate the boundary layer calculations, a refined mesh consisting of layers with prism elements was created in the vicinity of the heat exchanger walls. After grid dependence tests, the average number of mesh elements was about 0.9 million elements. For all cases studied, laminar flow, steady state conditions are set.

Boundary conditions:

- inlet condition: the inlet temperature and velocity were determined and the entrance flow velocity was assumed to be uniform,

- outlet condition: the pressure of fluid at the outlet section was set,

- at the upper and lower walls of channel: a constant temperature is set, with no-slip condition and no thickness,

- left and right sides of channel: a periodic boundary condition is set.

To solve the equations of the physical model, the variable-separation and implicit methods with second order scheme are used. In order to obtain a stable convergence and to decrease the occupation of machine resource, the SIMPLE (Semi Implicit Method for Pressure Linked Equation) algorithm was used for the velocity-pressure coupling. The residual target for all equations was set to $10^{-6}$. Calculations were performed on a machine with Intel Core i7 CPU, 2.20 $\mathrm{GHz}$ of clock speed and 8.0 GB of RAM. The convergence was obtained after about 1200-1500 iterations and from 5 to $6 \mathrm{~h}$ of $\mathrm{CPU}$ computational time.

\section{VALIDATION}

In this section, we present results of the validation of some numerical predictions. The reliability of the calculation code and the good choice of the numerical method and the boundary conditions are tested. For this purpose, reference was made to the experimental work done by Islamoglu [26]. With the same operating conditions undertaken by this author, the results of the Nusselt number are presented in Fig. 3 as a function of 
Reynolds number. As this figure shows, the comparison of the two results (numerical and experimental) shows a satisfactory agreement.

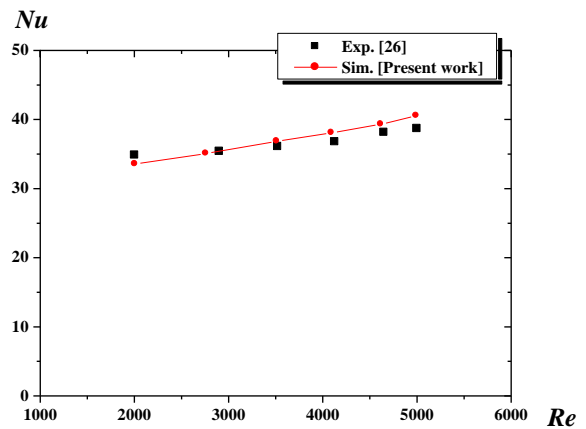

Fig. 3. Nusselt number vs. Reynolds number (validation)

\section{RESULTS AND DISCUSSION}

\subsection{Effect of the shape of corrugations}

The introduction of wall corrugation in heat transfer surfaces is one of the efficient techniques to enhance the heat transfer execution. As reported by [27], the corrugated channel heat exchangers, which are known by their high thermal efficiency, continue to replace the shell-tube heat exchangers in many applications. The flow fields through the corrugated channel are complex and characterized by the presence of secondary swirling flows known as Goertler vortices [28]. The heat transfer is controlled by the local flow structure.

The performance of PHEs are influenced by the number, shape and pattern of corrugations imprinted onto the channel walls. The corrugation design yields a separation and reattachment of flows, resulting thus in restarted boundary layer and enhanced heat transfer. However, the enhancement of heat transfer in this kind of heat exchangers is accompanied by a considerable amount in pressure drop. So, the Reynolds number should be lower in order to have an acceptable pressure drop. Moreover, $R e$ should not exceed the limit imposed by the onset of flooding $(R e<2000)$ if this heat exchanger is used a reflux condenser, as reported by Kanaris [29].

The shape of the corrugated wall can certainly play major roles in the thermo-hydraulic performance of a heat exchanger. Here, we explore the influence of three geometric configurations, namely: the semicircular, triangular and rectangular shapes.

The flow fields along the horizontal plane are presented on Fig. 4. The fluid particles undergo through a series of periodic passages (changes of directions: peaks and valleys). As observed on this figure, the hydrodynamics generated is periodic and the fluid velocity is accentuated at the end of the undulation (for circular and triangular shapes). For the rectangular shape, the velocity reaches its maximum on the first outgoing corner, and then it begins to gradually decrease to become neglected in immediate contact with the wall.

The viscous friction with the wall and the geometrical shape of the corrugation participate in the swirling formation behind the undulation. The triangular shape leads to the formation of a vortex spread over the channel length (i.e. the attachment length is greater for this undulation shape than for other cases). On the other hand, the great height of this vortex is observed for the "rectangular" case. While, the circular shape allows a rounding of the flow. Note that the height and length of these vortices depend greatly on the height and length of the undulation as will be discussed below.

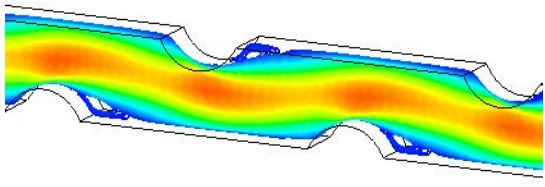

Circular shape

$1.670 \mathrm{e}-001$

1.471e-001

$-1.263 \mathrm{e}-001$

$-1.055 \mathrm{e}-001$

$8.473 \mathrm{e}-002$

$-6.394 \mathrm{e}-002$

4.315- -002

$2.237 \mathrm{e}-002$

$1.579 \mathrm{e}-003$

$-1921+-002$ $\left[\mathrm{m} \mathrm{s} \mathrm{s}^{\mathrm{s}-1]}\right.$

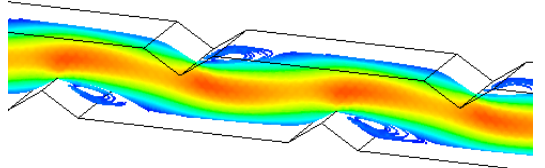

Triangular shape

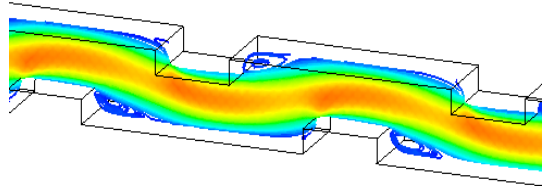

Rectangular shape

Fig. 4. Flow fields for $R e=200, h / a=0.3$

Fig. 5 gives more insight into the hydrodynamics, where the axial velocity is followed along the center line (Fig. 5a), then over a distance between two consecutive undulations at a position close to the wall (Fig. 5b). The flow is periodic for the three cases studied, and the maximum intensity of velocity $u$ is developed by the rectangular shape.

Concerning the vortex formation, the negative values of velocity $u$ indicate the existence of this structure (Fig. 5b). The vortex interaction seems less intense for the circular case compared to other cases.

This affects thermal efficiency as shown in Fig. 6. The vertical shape of the obstacle (rectangular shape) seems more effective than the other cases which are almost similar in this respect. But, whatever the form of the corrugation, it remains useful in terms of improvement of heat exchange compared to a smooth wall exchanger (Fig. 7).

On the other hand and as soon as there is an obstacle, the losses will increase. Also, the more the striking surface with the fluid is vertical and poorly rounded, there will be a growth of pressure drops (Fig. 8). 

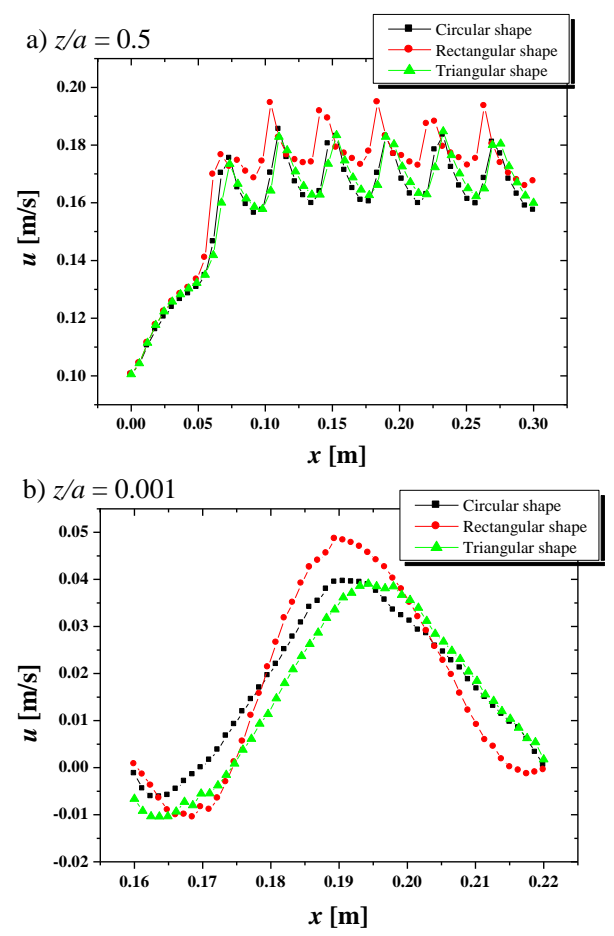

Fig. 5. Axial velocity for $R e=20, h / a=0.3, z / a=0.5$
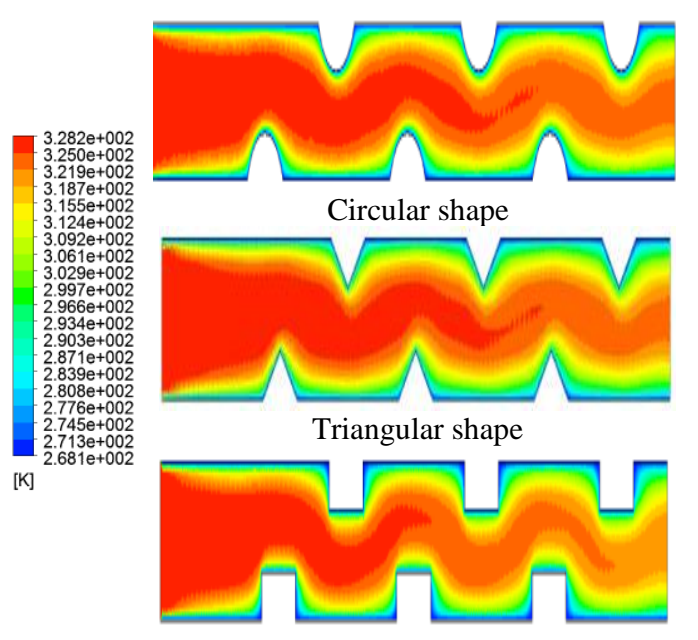

Rectangular shape

Fig. 6. Thermal field for $R e=20, h / a=0$

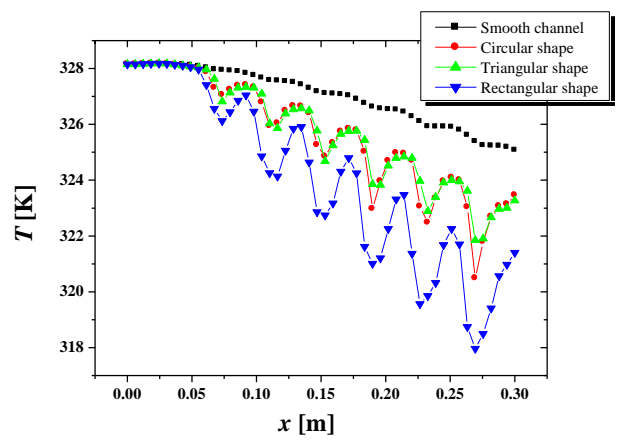

Fig. 7. Temperature distribution along the channel length, for $R e=20, h / a=0.3, z / a=0.5$

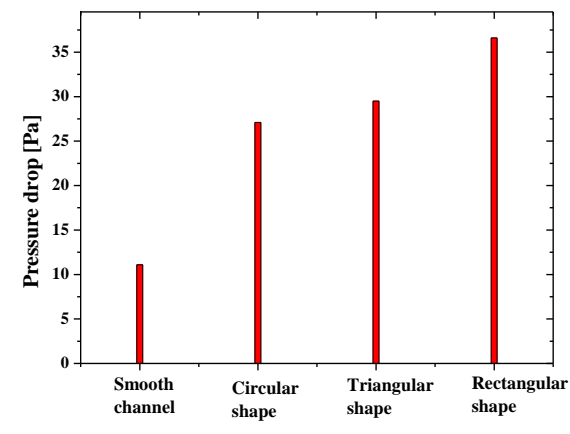

Fig. 8. Pressure drop for $R e=200, h / a=0.3$

\subsection{Effect of the number of corrugations}

In the second part of our investigation, we studied the effects of the number of undulations. Since the circular shape gave less pressure drop compared to other cases and improved heat exchange compared to the "smooth wall" case and a similarity to the "triangular" case, it was chosen for the rest of the our work.

Two cases are considered to perform the test concerning the number of undulations. The hydrodynamic structure is shown in Fig. 9 on a horizontal plane $X Y$. The speed is accentuated at the end of the ripple (Case No. 1), and it becomes even stronger with the increase of the number of ripples (Case No. 2). That means a presence of stronger molecular interaction, translated by the intensification of the vortices in Case No. 2.

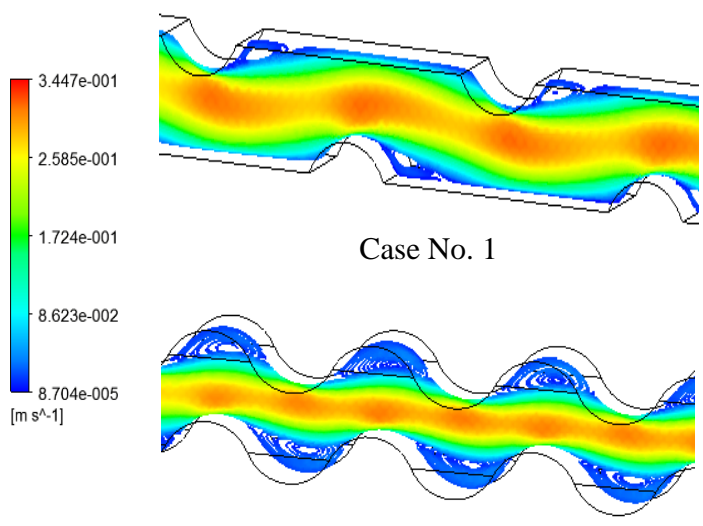

Case No. 2

Fig. 9. Streamlines for $R e=300, h / a=0.3$

The intensification of the molecular interaction created by Case No. 2 seems favorable in terms of heat exchange (Figs. 10 and 11). The increase in the number of corrugations is similar to an increase in the number of obstacles to the flow of hot fluid, which increases the residence time. But this is accompanied by an additional pressure drop (Fig. 12). 

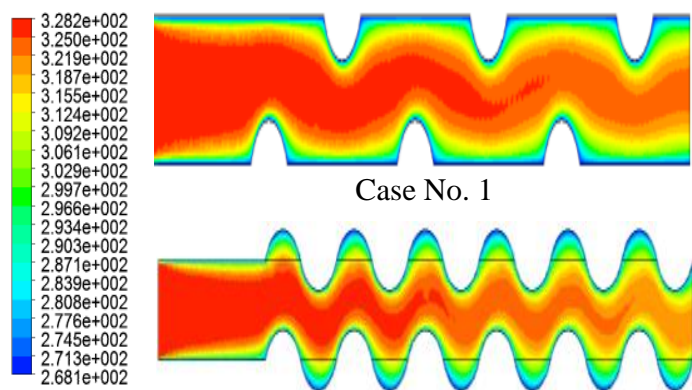

Case No. 1

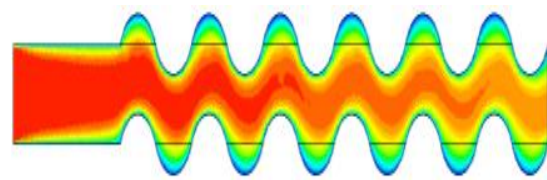

Case No. 2

Fig. 10. Thermal fields for $R e=20, h / a=0.3$

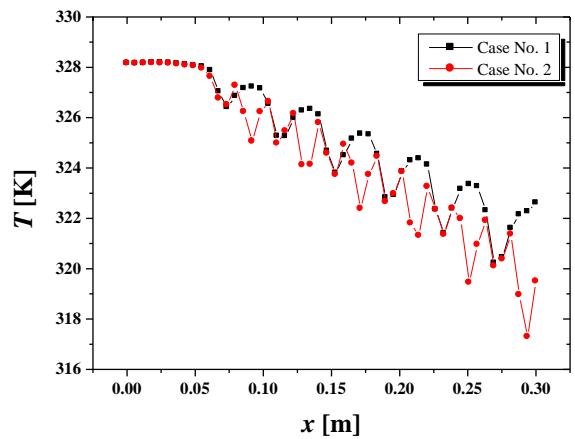

Fig. 11. Temperature distribution along the central axis of channel, $R e=20, h / a=0.3$

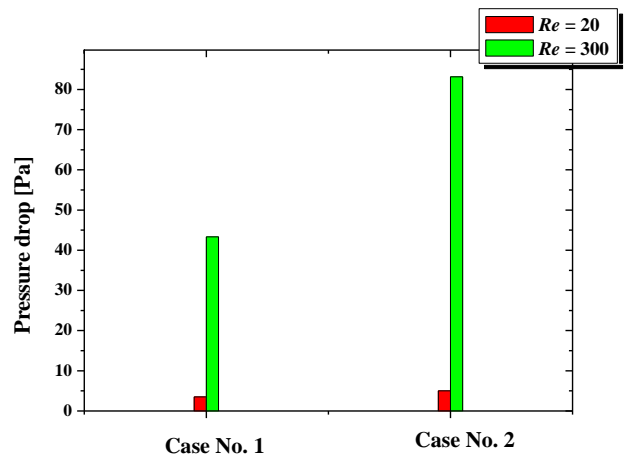

Fig. 12. Pressure drop for $h / a=0.3$

\subsection{Effect of the corrugation height}

The height of the undulations is another geometrical parameter that can justify its necessity to be optimized. Three cases are studied here: $h / a=0.2$, 0.3 and 0.4 . Hydrodynamics is well illustrated and in a variety of forms: streamlines (Fig. 13), curves (along the center line of the canal, Fig. 14a), curves (on the canal wall and along the distance between 2 undulations, Fig. 14b).

According to these results, the increase in the ratio $h / a$ makes the maximum speed increase at the end of the wave, the interaction between the particles is thus intensified and the size of the vortices is larger.
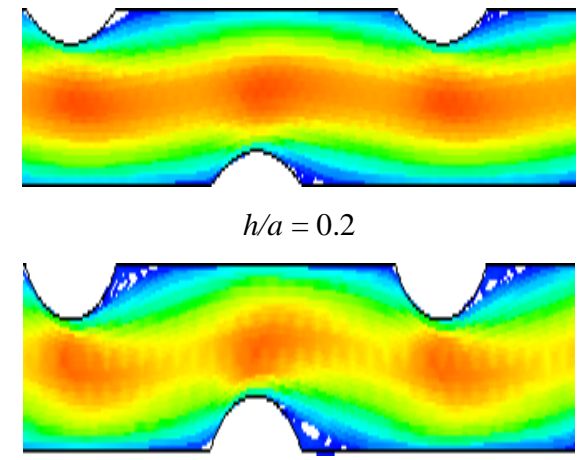

$h / a=0.3$

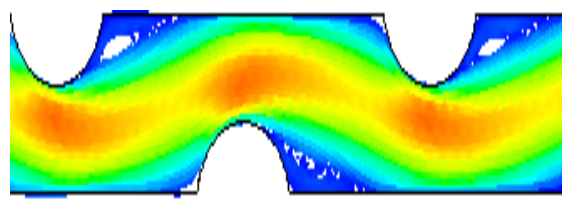

$$
h / a=0.4
$$

Fig. 13. Streamlines for $R e=200$, circular corrugation, Case No. 1

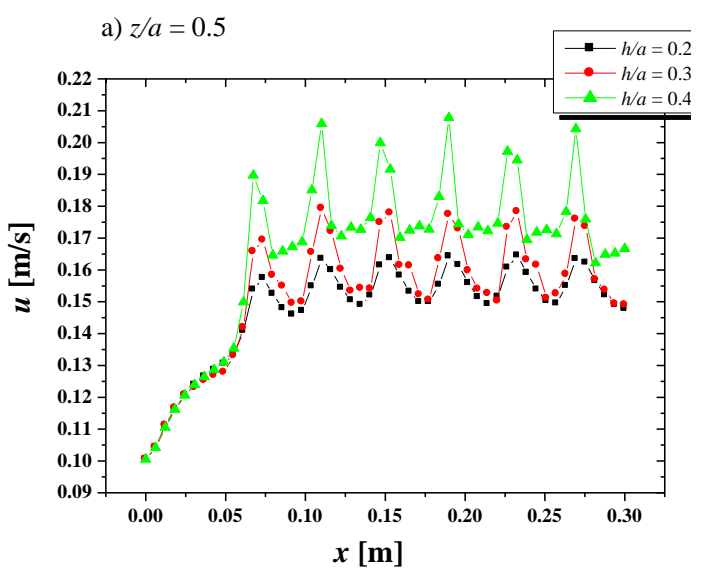

b) $\mathrm{z} / \mathrm{a}=0.001$

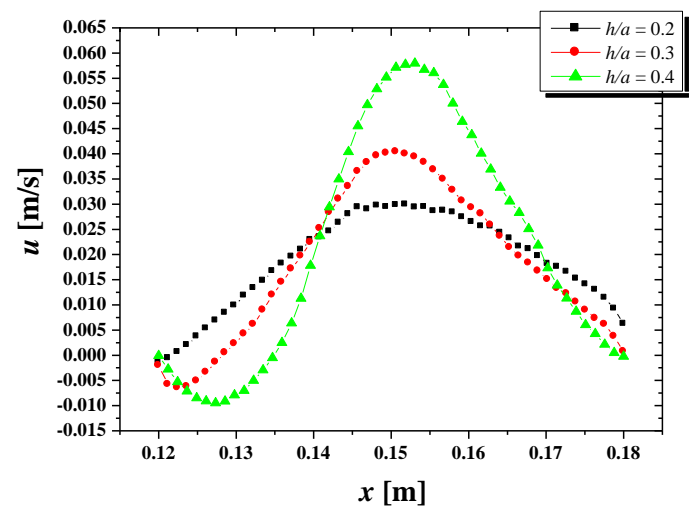

Fig. 14. Axial velocity along the channel for $R e=200$, circular corrugation, Case No. 1 

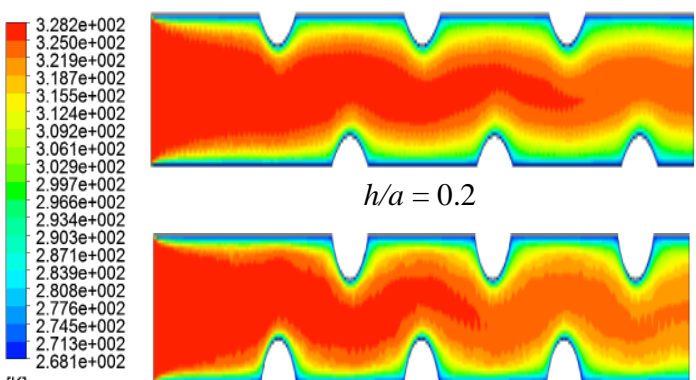

$h / a=0.2$

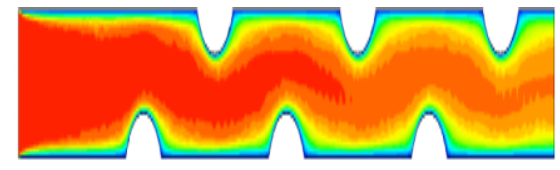

$h / a=0.3$

Fig. 15. Thermal fields for $R e=20$, circular corrugation, Case No.

The temperature distribution results are shown in Figs. 15 and 16. The growth of the ratio $h / a$ participates in the blockage of the fluid within the exchanger. This can allow heat removal over a short distance.

The increase of wall wave amplitude intensifies the flow separation bubble in the region near the corrugations and raises the turbulence intensity. The flow separation, recirculation, and reattachment interrupt the thermal boundary layer formed on the wall, therefore, the heat transfer ratio is increased.

In terms of pressure losses, and as expected, the ratio $h / a$ also has these effects on $\Delta P$. Proportionality is observed as shown in Fig. 17.

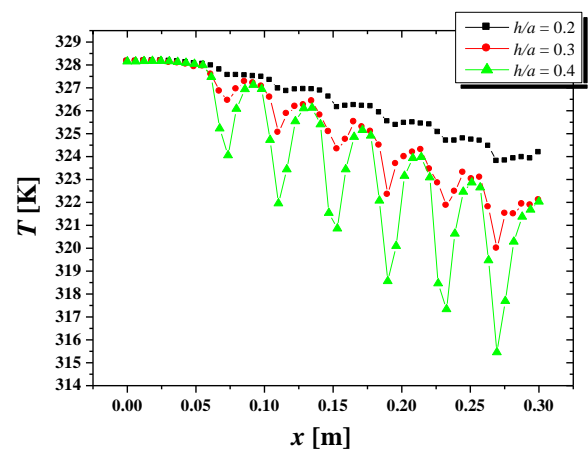

Fig. 16. Temperature distribution along the central axis of channel for $R e=20$, circular corrugation, Case No. 1

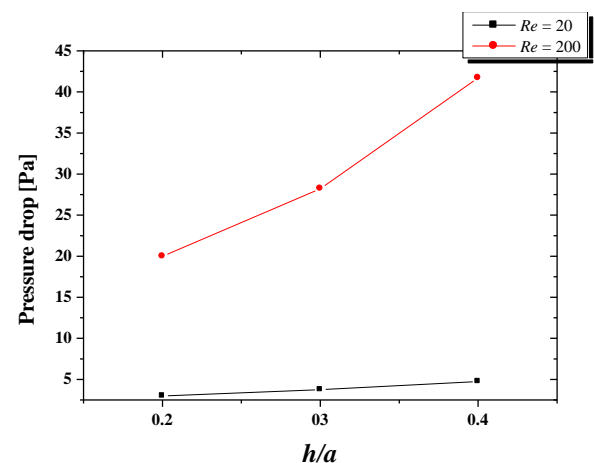

Fig. 17. Pressure drop for channel with circular corrugation, Case No. 1

\section{CONCLUSION}

A numerical investigation of the cooling of shear thinning fluids in a PHE has been performed. The technique of corrugated wall has been introduced to increase the heat transfer ratio. The performance of three shapes of corrugations have been compared, including the semi-circular, rectangular and triangular shapes. Also, effects of the number and height of corrugations have been explored.

Results of the numerical prediction showed the major advantage of the presence of corrugations on the heat exchange improvement. In terms of good heat transfer, the shapes of corrugations studied can be classified as follows: rectangular $>$ triangular $>$ semicircular. Then, in terms of reduced pressure drop, this ranking is reversed. We note that the triangular and semi-circular shapes have almost similar effects.

The increase of the number and height of corrugations is beneficial in terms of improved heat exchange, but with a continuous growth of pressure drops.

Finally, and concerning the Reynolds number, even if the increase of this parameter causes an intensification of turbulence and molecular interaction, this will create a faster flow than necessary and does not allow sufficient time for the hot fluid to be cooled. On the other hand, the pressure drops will be more considerable with the growth of $R e$ which is due to the increase of the viscous friction.

\section{References}

1. Sahel D., Ameur H., Benzeguir R., Kamla Y. (2016). Enhancement of heat transfer in a rectangular channel with perforated baffles. Applied Thermal Engineering, Vol. 101, pp. 156-164.

2. Boukhadia K., Ameur H., Sahel D., Bozit M. (2018). Effect of the perforation design on the fluid flow and heat transfer characteristics of a plate fin heat exchanger. International Journal of Thermal Sciences, Vol. 126, pp. $172-180$

3. Mellal M., Benzeguir R., Sahel D., Ameur H. (2017). Hydro-thermal shell-side performance evaluation of a shell and tube heat exchanger under different baffle arrangement and orientation. International Journal of Thermal Sciences, Vol. 121, pp. 138-149.

4. Medjahed D. M., Ameur H., Ariss A., Medjadji N., Mahammedi A. (2016). Heat transfer improvement in micro-channel heat sinks by modifying some design and operating conditions. International Review of Mechanical Engineering, Vol. 10, pp. 395-404.

5. Lin L., Zhao J., Lu G., Wang X.D., Yan W.M. (2017). Heat transfer enhancement in microchannel heat sink by changing wavelength/amplitude. International Journal of Thermal Sciences, Vol. 118, pp. 423-434.

6. Alem K., Sahel D., Nemdili A., Ameur H. (2018). CFD investigations of thermal and dynamic behaviors in a tubular heat exchanger with butterfly baffles. Frontiers in Heat and Mass Transfer (FHMT), Vol. 10, pp. 27.

7. Lin J.H., Huang C.Y., Su C.C. (2007). Dimensional analysis for the heat transfer characteristics in the 
corrugated channels of plate heat exchangers. International Communications in Heat and Mass Transfer, Vol. 34, pp. 304-312

8. Dong J.Q., Su L., Chen Q., Xu W.W. (2013). Experimental study on thermal-hydraulic performance of a wavy fin-and-flat tube aluminum heat exchanger. Applied Thermal Engineering, Vol. 51, pp. 32-39.

9. Ni M.L., Chen Y.P., Dong C., Wu. J.F. (2014). Numerical simulation of heat transfer and flow of cooling air in triangular wavy fin channels. Journal of Central South University of Technology, Vol. 21, pp. 2759-2765.

10. Rush T.A., Newell T.A., Jacobi A.M. (1999). An experimental study of flow and heat transfer in sinusoidal wavy passages. International Journal of Heat and Mass Transfer, Vol. 42, pp. 1541-1553.

11. Bahaidarah H.M., Anand N., Chen H. (2005). Numerical study of heat and momentum transfer in channels with wavy walls. Numerical Heat Transfer, Part A, Vol. 47, pp. 417-439.

12. Tokgoz N., Aksoy M.M., Sahin B. (2017). Investigation of flow characteristics and heat transfer enhancement of corrugated ducts geometries. Applied Thermal Engineering, Vol. 118, pp. 518-530.

13. Wang C.C., Chen C.K. (2002). Forced convection in a wavy-wall channel. International Journal of Heat and Mass Transfer, Vol. 45, pp. 2587-2595.

14. Blomerius H., Mitra N.K. (2000). Numerical investigation of convective heat transfer and pressure drop in wavy ducts. Numerical Heat Transfer, Part A, Vol. 37, pp. 37-54.

15. Ali A.H.H., Hanaoka Y. (2002). Experimental study on laminar flow forced-convection in a channel with upper $\mathrm{V}$-corrugated plate heated by radiation. International Journal of Heat and Mass Transfer, Vol. 45, pp. 21072117.

16. Mohammed H., Abed A.M., Wahid M. (2013). The effects of geometrical parameters of a corrugated channel with in out-of-phase arrangement. International Communications in Heat and Mass Transfer, Vol. 40, pp. 47-57.

17. Taymaz I., Koc I., Islamoglu Y. (2008). Experimental study on forced convection heat transfer characteristics in a converging diverging heat exchanger channel. Heat and Mass Transfer, Vol. 44, pp. 1257-1262.

18. Ma T., Du L.X., Sun N., Zeng M., Sunden B., Wang Q.W. (2016). Experimental and numerical study on heat transfer and pressure drop performance of Cross-Wavy primary surface channel. Energy Conversion and Management, Vol. 125, pp. 80-90.

19. Wang L., Deng L., Ji C., Liang E., Wang C., Che D. (2016). Multi-objective optimization of geometrical parameters of corrugated-undulated heat transfer surfaces. Applied Energy, Vol. 174, pp. 25-36.

20. Bilen K., Cetin M., Gul H., Balta T. (2009). The investigation of groove geometry effect on heat transfer for internally grooved tubes. Applied Thermal Engineering, Vol. 29, pp. 753-761.

21. Jialing Z., Yupei W., Wei Z. Xueling L. (2014). Numerical study on heat transfer enhancement for use of corrugated, nodal and horizontal grain tubes. Transactions of Tianjin University, 2014, 20: 385-392.

22. Elshafei E.A.M., Awad M.M., El-Negiry E., Ali A.G. (2010). Heat transfer and pressure drop in corrugated channels. Energy, Vol. 35, pp. 101-110.

23. Gao X.M., Li W.Y. Wang J.S. (2014). Heat transfer and flow characteristics in a channel with one corrugated wall. Science China Technological Sciences, Vol. 57, pp. 2177-2189.

24. Zhang L., Che D. (2011). Influence of corrugation profile on the thermal-hydraulic performance of cross- corrugated plates. Numerical Heat Transfer, Part A, Vol. 59, pp. 267-296.

25. Akbarzadeh M., Rashidi S., Esfahani J.A. (2017). Influences of corrugation profiles on entropy generation, heat transfer, pressure drop and performance in a wavy channel. Applied Thermal Engineering, Vol. 116, 278291.

26. Islamoglu Y. (2008). Effect of rounding of protruding edge on convection heat transfer in a converging diverging channel. International Communications in Heat and Mass Transfer, Vol. 35, pp. 643-647.

27. Kays W.M., London A.L. (1998). Compact heat exchangers. $3^{\text {rd }}$ ed., Krieger Publ. Co., Melbourne, Florida.

28. Hesselgraves J.E. (2001). Compact heat exchangers: selection, design and operation, $1^{\text {st }}$ ed., Pergammon, New York.

29. Kanaris A.G., Mouza A.A., Paras S.V. (2006). Flow and heat transfer prediction in a corrugated plate heat exchanger using a CFD code. Chemical Engineering and Technology, Vol. 29, pp. 923-930.

\section{Biographical notes}

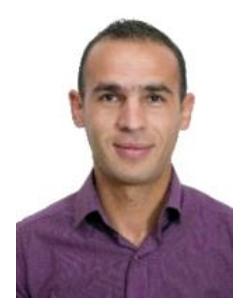

Houari Ameur is a Doctor in the Department of Technology at University Center of Naama (Ctr Univ Naama). He received his Engineering Diploma in 2006 in Marine Engineering, his Master Degree in 2008, his $\mathrm{PhD}$ in 2012 and his HDR in 2013 in Mechanical Engineering from University of Science and Technology, Oran (USTOMB), Algeria. His current research interests are mainly in the area of non-Newtonian fluid flows, mechanical mixing, Computational Fluid Dynamics, heat exchangers and thermal applications.

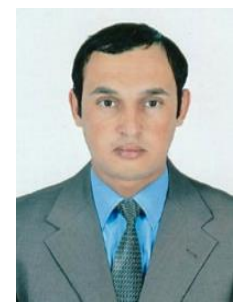

Djamel SAHEL is a Doctor in the Depatment of Technical Sciences at University of Laghouat, Algeria. He obtained his engineering degree in energy mechanics in 2007. In the same specialty, he obtained his Master and $\mathrm{PhD}$ degrees in 2011 and 2017, respectively from the University of Science and Technology USTO-MB, Oran, Algeria. Her research fields are heat transfer, fluid flow, membrane systems and optimization of thermal systems. 